University of Nebraska - Lincoln

DigitalCommons@University of Nebraska - Lincoln

\title{
Resonances and threshold effects in low-energy electron collisions with methyl halides
}

Gordon A. Gallup

University of Nebraska-Lincoln, ggallup1@unl.edu

Ilya I. Fabrikant

University of Nebraska-Lincoln, ifabrikant@unl.edu

Follow this and additional works at: https://digitalcommons.unl.edu/physicsgallup

Part of the Physics Commons

Gallup, Gordon A. and Fabrikant, llya I., "Resonances and threshold effects in low-energy electron collisions with methyl halides" (2007). Gordon Gallup Publications. 44.

https://digitalcommons.unl.edu/physicsgallup/44

This Article is brought to you for free and open access by the Research Papers in Physics and Astronomy at DigitalCommons@University of Nebraska - Lincoln. It has been accepted for inclusion in Gordon Gallup Publications by an authorized administrator of DigitalCommons@University of Nebraska - Lincoln. 


\title{
Resonances and threshold effects in low-energy electron collisions with methyl halides
}

\author{
Gordon A. Gallup and Ilya I. Fabrikant \\ Department of Physics and Astronomy, University of Nebraska, Lincoln, Nebraska 68588-0111, USA
}

(Received 2 January 2007; published 26 March 2007)

\begin{abstract}
Cross sections for elastic and inelastic electron collisions with $\mathrm{CH}_{3} X(X=\mathrm{Cl}, \mathrm{Br}, \mathrm{I})$ molecules are calculated. For the lowest partial wave, the resonance $R$-matrix theory, and for the higher partial waves, the theory of scattering by dipolar plus polarization potential, are used. It is shown that the rotationally elastic scattering amplitude for a polar molecule in the fixed-nuclei approximation is logarithmically divergent for the forward direction, and a closure formula is derived to speed up the convergence at small angles. In treating the nuclear motion, only $\mathrm{C}-X$ stretch vibrations are taken into account. The dipole moment as a function of the $\mathrm{C}-X$ distance is modeled by a function incorporating the experimental value of the molecular dipole moments at the equilibrium distance and the derivatives of the dipole moments extracted from the experimental data on infrared intensities. This is supplemented by ab initio calculations of the dipole moment function for $\mathrm{CH}_{3} \mathrm{Br}$ using the multiconfigurational valence bond method. The results for scattering cross sections show pronounced features caused by vibrational Feshbach resonances and threshold cusps. The features are most noticeable at the $v=6,7$, and 8 thresholds in $\mathrm{CH}_{3} \mathrm{Cl}$, at the $v=3$ and 4 thresholds in $\mathrm{CH}_{3} \mathrm{Br}$, and at the $v=1$ threshold in $\mathrm{CH}_{3} \mathrm{I}$.
\end{abstract}

DOI: 10.1103/PhysRevA.75.032719

\section{INTRODUCTION}

The recent development of highly resolved-in-energy electron sources [1-4] has stimulated further experimental and theoretical studies of low-energy electron collisions with polyatomic molecules [5-14]. Other advances in experimental research have enhanced the ability to study the rates of collisional processes for selectively excited vibrational levels [15-17].

If the long-range electron-molecule interaction is strong enough, it can support vibrational Feshbach resonances (VFRs), a special scattering resonance with the electron weakly bound to a vibrationally excited state of the neutral molecule $[18,19]$. These resonances have been observed in $\mathrm{HF}$ [20,21], $\mathrm{CH}_{3} \mathrm{I}$ [6], $\mathrm{CH}_{2} \mathrm{Br}_{2}$ [9], $\mathrm{N}_{2} \mathrm{O}$ [22], and molecular clusters $[5,10]$. It is customary to relate VFRs to the electron interaction with the molecular dipole moment, but quite often (for example, in the case of $\mathrm{CH}_{3} \mathrm{I}$ [6]), VFR appears due to the combination of the dipolar and polarization interactions. It is becoming apparent that VFRs are a quite common phenomenon. Recent experiments indicate their importance in biologically relevant molecules, particularly uracil and thymine $[23,24]$. They also play an important role in positron-molecule scattering, in particular, enhancing positron annihilation $[25,26]$.

In some systems the electron-molecule interaction is not strong enough to support a weakly bound state, but is close to producing it. In this case virtual-state related cusps are observed at the vibrational excitation thresholds, particularly in $\mathrm{CF}_{3} \mathrm{I}$ [12] and $\mathrm{CF}_{3} \mathrm{Br}$ [13] molecules.

Most experimental information on VFRs and dipolesupported cusps has been extracted from dissociative electron attachment (DEA) measurements. Much less is known how these phenomena show up in elastic electron scattering and vibrational excitation (VE). Two joint experimental and theoretical studies illustrate this connection for $\mathrm{CH}_{3} \mathrm{I}[6,27]$. The permanent dipole moment of the $\mathrm{CH}_{3} \mathrm{I}$ molecule is rather small, $D=0.639$ a.u., and the infrared activity is rather weak (the transition dipole moment is about 0.015 a.u.). For
PACS number(s): 34.80.Bm, 34.80.Gs, 34.80.Ht, 34.60.+z

other methyl halides these quantities are higher, therefore the contribution of direct scattering, both in elastic and VE channels, becomes important. In particular it is of interest to know how direct and resonant contributions interfere in VE cross section. For detailed investigation of this problem the dipole moment as a function of nuclear geometry $\mu(R)$ is necessary. This dependence, for example, can significantly affect threshold peaks in vibrational excitation of hydrogen halides [28].

In the present work we study low-energy electron collisions with $\mathrm{CH}_{3} X$, where $X$ stands for $\mathrm{Cl}, \mathrm{Br}$, and $\mathrm{I}$, with the inclusion of only $\mathrm{C}-X$ symmetric stretch vibrations. Previous theoretical studies $[6,7,29,30]$ showed that this approximation is adequate for theoretical description of electron collisions with these compounds. Therefore we are interested in the dipole moment as a function of the $\mathrm{C}-X$ distance. We calculate first the dipole moment function for $\mathrm{CH}_{3} \mathrm{Br}$ and compare the result with a model based on experimental values of the equilibrium dipole moment [31] and the transition dipole moment extracted from the infrared intensities data [32]. We demonstrate then that DEA cross sections are very close for both dipole moment functions, and extend our calculations to $\mathrm{CH}_{3} \mathrm{Cl}$ and $\mathrm{CH}_{3} \mathrm{I}$. The cross sections exhibit pronounced threshold structures in VE and elastic cross sections, but these features become weaker for very weakly bound states.

\section{THEORY}

For cross section calculations we employ the one-pole $R$-matrix theory [33] with one active $\mathrm{C}$ - $X$ stretching mode which has been successfully applied to description of DEA to $\mathrm{CH}_{3} \mathrm{Cl}[29,30], \mathrm{CH}_{3} \mathrm{I}[6,7]$, and $\mathrm{CH}_{3} \mathrm{Br}$ [7] molecules. We modify it by inclusion of both more accurate dipole moment functions and direct contributions to elastic scattering and VE. These modifications are described below.

\section{A. Dipole moment function}

In our previous DEA calculations $[6,7,29,30]$ for methyl halides $\mathrm{CH}_{3} \mathrm{X}$ we employed the dipole moment functions 
TABLE I. Some data for the methyl radical.

Number of electrons

Multiplicity

Dipole moment

2

0.2258 a.u.

$\mu(R)$ calculated for hydrogen halides $\mathrm{HX}$ [34], but rescaled them to incorporate the experimental value of the dipole moment at the equilibrium internuclear separation. Specifically, we used the following Padé approximant for $\mu(R)$ :

$$
\mu(R)=\mu_{0} \frac{(1+x)^{3}}{1+\sum_{n=1}^{7} c_{n} x^{n}},
$$

where $x=\left(R-R_{e}\right) / R_{e}$ and $R_{e}$ is the equilibrium internuclear separation.

This approach can be modified further to take into account the observed intensities in the infrared spectra [32]. They generate the transition dipole moment $\mu_{01}$, and this can be used to calculate $\mu^{\prime}$, the derivative of the dipole moment in $x$ at $x=0$ :

$$
\mu^{\prime}=\sqrt{2 M \omega} R_{e} \mu_{01},
$$

where $M$ is the reduced mass, and $\omega$ is the vibrational frequency. $\mu^{\prime}$ generates the coefficient $c_{1}$ in expansion (1).

To compare the model dipole moment function with $a b$ initio results, we performed multiconfiguration valence bond (MCVB) $[35,36]$ calculation of $\mathrm{CH}_{3} \mathrm{Br}$ to determine the dependence of the electric dipole moment on the $\mathrm{C}-\mathrm{Br}$ distance. All of the calculations were done with a 6-31G(d) (GAMESS [37] definition) Gaussian basis set.

The MCVB formulation appropriate for this problem involves a prior calculation of the spin-restricted open-shell Hartree-Fock (ROHF) structures of $\mathrm{CH}_{3}$ and $\mathrm{Br}$ separately. Although it would be possible to do the calculations at each $\mathrm{C}$-Br distance with the methyl group in its corresponding equilibrium geometry, in this case we used a rigid geometry corresponding to the angles present in the equilibrium geometry of the whole $\mathrm{CH}_{3} \mathrm{Br}$ molecule. Additional calculations with relaxed geometry show that qualitatively $\mathrm{H}$ atoms nearly follow the $\mathrm{C}$ atom during the vibration. We conclude that the approximation of the fixed $\mathrm{CH}_{3}$ geometry should not alter the results significantly. Table I gives some values obtained in our calculations for the methyl radical. The configuration (in $C_{3 v}$ ) is $1 a_{1}^{2} 2 a_{1}^{2} 1 e^{4} 3 a_{1}$ and the singly occupied orbital $3 a_{1}$ is essentially an $s p^{3}$ hybrid pointing away from the $\mathrm{H}$ atoms.

The $\mathrm{Br}$ atom was also treated alone in the conventional way with an open shell Roothaan treatment. It is also a doublet system in a ${ }^{2} A_{1}$ state when considered in $C_{3 v}$ symmetry. The configuration we used is

$$
\text { Ar: } 3 d^{10} 4 s^{2} 4 p^{5}
$$

and it has 35 electrons. The singly occupied orbital is essentially of $4 p_{z}$ character.

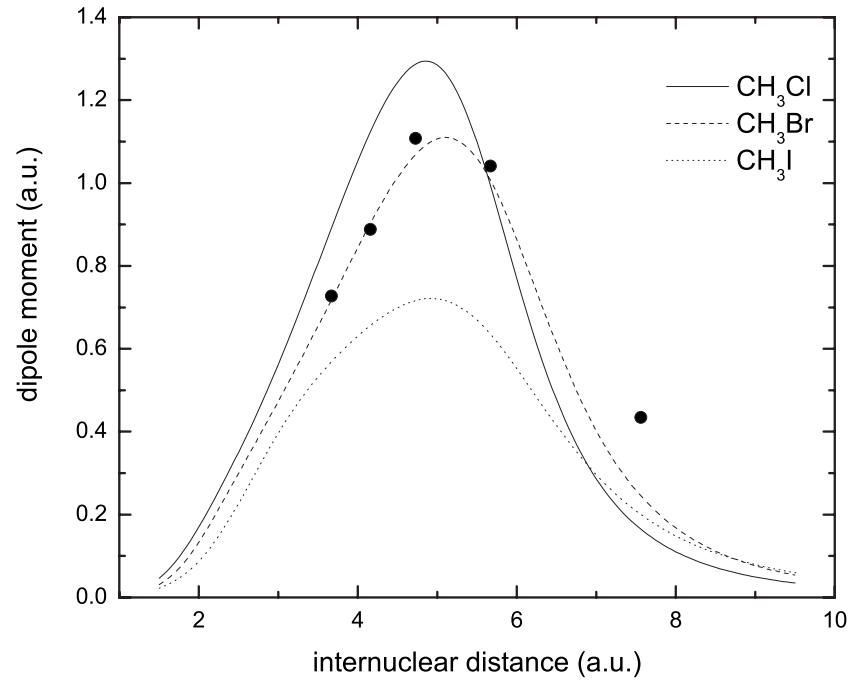

FIG. 1. Dipole moment as a function of $\mathrm{C}-\mathrm{X}$ distance for $\mathrm{CH}_{3} \mathrm{X}$ compounds. Lines: model function, see text. Circles: MCVB calculations.

All together, $\mathrm{CH}_{3} \mathrm{Br}$ has 44 electrons, and the MCVB calculations involved dividing the electrons into two groups, the "inner" set of 30 electrons arranged to generate a static exchange potential for the remaining 14, which were arranged in various occupations of the "outer" orbitals. The configurations included follow the pattern used successfully before with single and double "excitations" out of a set of 36 "ground state" (reference state) configuration functions. With the symmetry constraints 780 terms were produced.

Figure 1 shows the model dipole functions for all methyl halides and comparison with the MCVB calculations for $\mathrm{CH}_{3} \mathrm{Br}$ described above. The overall agreement between two functions is quite good. Our scattering calculations show that the difference between the two dipole functions becomes important only for highly excited vibrational states. To illustrate this we present in Fig. 2 DEA cross sections for $v=7$ and $v=11$ states, in the energy region where the difference is most noticeable. Here $v$ stands for the vibrational quantum number of the symmetric stretch $\left(\nu_{3}\right)$ mode. Even for $v=11$ the difference is not substantial. The step structures are associated with vibrational excitation thresholds.

All results presented in the rest of the paper have been calculated with the model dipole moment function, Eq. (1).

\section{B. Vibrational excitation cross section}

We will assume that the molecule has a fixed orientation during the collision and that the projection of the electron angular momentum on the molecular axis $m$ is a good quantum number. The first approximation works well for energies exceeding the rotational spacing. The second assumption, strictly speaking, does not apply to nondiatomic molecules, but it can be justified by noticing that the low-energy resonant scattering by methyl halides is dominated by the $\sigma^{*}$ resonance, and for the pure dipole scattering $m$ is conserved. We will also assume that the angular part of the electron wave function in the outer region is a dipolar angular harmonic satisfying the equation $[38,39]$ 


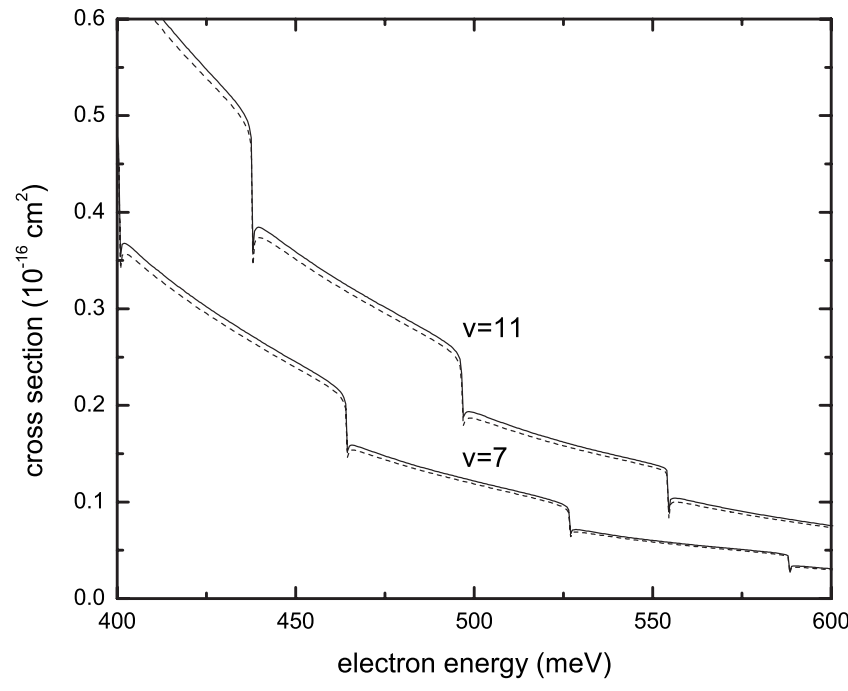

FIG. 2. DEA to vibrationally excited states of $\mathrm{CH}_{3} \mathrm{Br}$. Solid lines: calculation with the dipole function from the MCVB calculations. Dashed lines: calculation with the model dipole function.

$$
\begin{gathered}
{\left[\nabla_{\theta \phi}^{2}+2 \mu_{v v} \cos \theta+\lambda_{\nu}^{m}\left(\lambda_{\nu}^{m}+1\right)\right] Z_{\nu}^{m}(\theta, \phi)=0,} \\
\nu=m, m+1, \ldots, \\
Z_{\nu}^{m}(\theta, \phi)=D_{\nu}^{m}(\cos \theta) e^{i m \phi},
\end{gathered}
$$

where $\nabla_{\theta \phi}^{2}$ is the angular part of the Laplace operator and $\mu_{v v}$ is the dipole moment for a given vibrational state $v$. Then the amplitude for vibrational transition from $v$ state to $v^{\prime}$ state is

$$
\begin{aligned}
f_{v v^{\prime}}\left(\xi_{0}, \xi\right)= & -\frac{2 i \mu_{v v^{\prime}}}{q} \cos \gamma \\
& -\frac{i}{\left(k k^{\prime}\right)^{1 / 2}} S_{v v^{\prime}} D_{0}^{0}\left(-\cos \xi_{0}\right) D_{0}^{0}(\cos \xi),
\end{aligned}
$$

where $\xi_{0}$ and $\xi$ are incident and scattering angles relative to the molecular axis, $\mathbf{k}$ and $\mathbf{k}^{\prime}$ are initial and final electron momenta, $\mathbf{q}=\mathbf{k}^{\prime}-\mathbf{k}, \gamma$ is the angle between $\mathbf{q}$ and the molecular axis, $\mu_{v v^{\prime}}$ is the matrix element of the dipole moment, and $S_{v v^{\prime}}$ is the $S$-matrix element for the resonance vibrational excitation. The first term in Eq. (5) represents the direct excitation in the Born approximation [40] and the second the resonance contribution [41]. Since we will be mostly interested in the transition $v \rightarrow v+1$, we neglect the difference in the diagonal dipole moment in initial and final state, and assume that the dipolar harmonic $D_{0}^{0}$ is the same for both states.

Taking the square of the absolute value of the amplitude and averaging over orientations, we obtain for the differential cross section as a function of the scattering angle $\theta$

$$
\sigma(\theta)=\frac{k^{\prime}}{k}\left[A_{\text {dir }}(\theta)+A_{\text {res }}(\theta)+A_{\text {int }}(\theta)\right]
$$

where $A_{d i r}(\theta)=4 \mu_{v v^{\prime}}^{2} / 3 q^{2}(\theta)[40]$. The method of calculation of the resonance contribution $A_{\text {res }}$ was described elsewhere [41]. Here we will concentrate on the interference term $A_{\text {int }}$,

$$
A_{i n t}=\frac{4 \mu_{v v^{\prime}} \operatorname{Re} S_{v v^{\prime}}}{q\left(k k^{\prime}\right)^{1 / 2}}\left\langle\cos \gamma D_{0}^{0}\left(-\cos \xi_{0}\right) D_{0}^{0}(\cos \xi)\right\rangle,
$$

where angular brackets mean the average over orientations. To calculate the average, we write

$$
\cos \gamma=\frac{\mathbf{k}^{\prime} \cdot \hat{\mathbf{s}}-\mathbf{k} \cdot \hat{\mathbf{s}}}{q},
$$

where $\hat{\mathbf{s}}$ is a unit vector in the direction of the molecular axis. Then we expand $D_{0}^{0}(\cos \xi)$ in Legendre polynomials and use the addition theorem for spherical harmonics:

$$
\begin{aligned}
D_{0}^{0}(\cos \xi) & =\sum_{l}\left(\frac{2 l+1}{2}\right)^{1 / 2} a_{l} P_{l}(\cos \xi) \\
& =2 \pi \sum_{l m}\left(\frac{2}{2 l+1}\right)^{1 / 2} a_{l} Y_{l m}^{*}(\hat{\mathbf{k}}) Y_{l m}(\hat{\mathbf{s}}),
\end{aligned}
$$

where we have performed transformation to the frame with the polar axis along the vector $\mathbf{k}$. The expansion coefficients $a_{l}$ can be easily obtained by the diagonalization of Eq. (3). Using a similar expansion for $D_{0}^{0}\left(-\cos \xi_{0}\right)$ and integrating over $\hat{\mathbf{s}}$, we obtain

$$
\left\langle\cos \gamma D_{0}^{0}\left(-\cos \xi_{0}\right) D_{0}^{0}(\cos \xi)\right\rangle=\frac{k+k^{\prime}}{q} \sum_{l} p_{l} P_{l}(\cos \theta),
$$

$$
p_{l}=\frac{a_{l}(-1)^{l}}{(2 l+1)^{1 / 2}}\left[\frac{l+1}{(2 l+3)^{1 / 2}} a_{l+1}+\frac{l}{(2 l-1)^{1 / 2}} a_{l-1}\right] .
$$

Now the interference term can be written as

$$
A_{\text {int }}(\theta)=\frac{2 \mu_{v v^{\prime}}}{q^{2}\left(k k^{\prime}\right)^{1 / 2}}\left(k+k^{\prime}\right) \operatorname{Re} S_{v v^{\prime}} \sum_{l} p_{l} P_{l}(\cos \theta) .
$$

The interference term integrated over scattering angle can be obtained using the equation

$$
\int_{-1}^{1} \frac{P_{l}(x)}{k^{2}+\left(k^{\prime}\right)^{2}-2 k k^{\prime} x} d x=\frac{1}{k k^{\prime}} Q_{l}\left(\frac{k^{2}+\left(k^{\prime}\right)^{2}}{2 k k^{\prime}}\right),
$$

where $Q_{l}(x)$ is the Legendre function of the second kind.

Finally the integrated cross section is

$$
\begin{aligned}
\sigma_{v v^{\prime}}= & \frac{\pi}{k^{2}}\left\{\frac{8 \mu_{v v^{\prime}}^{2}}{3} \ln \frac{k+k^{\prime}}{\left|k-k^{\prime}\right|}+\left|S_{v v^{\prime}}\right|^{2}\right. \\
& \left.+4 \mu_{v v^{\prime}} \frac{k+k^{\prime}}{\left(k k^{\prime}\right)^{1 / 2}} \operatorname{Re} S_{v v^{\prime}} \sum_{l} p_{l} Q_{l}\left(\frac{k^{2}+\left(k^{\prime}\right)^{2}}{2 k k^{\prime}}\right)\right\} .
\end{aligned}
$$

Note that the phase factor in $\mu_{v v^{\prime}}$ should be consistent with the phase factor in $S_{v v^{\prime}}$, since the sign of the interference term is determined by the product $\mu_{v v^{\prime}} \operatorname{Re} S_{v v^{\prime}}$.

\section{Differential elastic cross section}

It is well known that resonant features in elastic electron scattering by polar molecules can be significantly suppressed 
by the strong direct contribution. Moreover, in the approximation of a fixed molecular orientation the total elastic cross section is divergent. For diatomic polar molecules in $\Sigma$ electronic states and asymmetric tops this divergence can be removed by inclusion of rotations for large orbital angular momentum of the incident electron (or small scattering angles) and calculation of the corresponding scattering amplitudes in the Born dipole approximation [39]. However, for symmetric top molecules, such as methyl halides, even inclusion of rotations does not average out the permanent dipole moment, and the total cross section is still divergent [42]. The reason for this is the presence of degenerate channels coupled by the dipolar interaction $[43,44]$. To remove this degeneracy, the inversion splitting should be included [45], and the integrated cross section becomes proportional to the logarithm of the inversion splitting.

In a series of molecules such as those in this study, the inversion splitting is very difficult to determine. About all one can say about it is that it must be an exceedingly small energy difference. The actual motion over the inversion barrier can be viewed as some linear combination of the $A_{1}$ normal modes of the harmonic vibrations, but, of course, when inversion splitting is included, the nuclei are no longer treatable as classical particles. Thus, there is a sense in which the whole system must be considered to have $D_{3 h}$ symmetry. In addition to this complication, a correct treatment would require the handling of effects of whatever nuclear spin states are present. The upshot is that the extremely small energy difference will produce a total elastic cross section that might as well be infinite, as far as any experiment is concerned. Since the range of impact parameters contributing to the total cross section becomes enormous, we do not think that it is of much practical value to calculate the total cross section. Since most of the available experimental data on elastic scattering by polar symmetric tops were obtained for angle-differential cross sections, we are calculating these in the present paper.

We base our description of elastic scattering by a polar molecule on Refs. [41,46,47]. The differential cross section for a diatomic molecule can be decomposed in partial cross sections for $J^{\prime} M^{\prime} \rightarrow J M$ transitions, where $J^{\prime} M^{\prime}$ and $J M$ are the rotational quantum number and its projection in the initial and final states, respectively. In the fixed-nuclei approximation the cross section summed over $J M$ and averaged over $M^{\prime}$ does not depend on the initial rotational quantum number $J^{\prime}$ [48]. Therefore a nondiatomic molecule in the fixed-nuclei approximation can be treated as a diatomic with fictitious quantum numbers $J^{\prime} M^{\prime}$. Because the independence of $J^{\prime} M^{\prime}$ has been proven, we will use for simplicity $J^{\prime}=M^{\prime}=0$.

We will describe elastic scattering by the $S$ matrix in the dipolar harmonics representation given by functions (3). We assume that at $(\nu, m)=(0,0)$ the elastic scattering is described by the matrix element $S_{00}$ calculated by the $R$-matrix method which includes resonance, dipole and polarization contributions. For $(\nu, m) \neq(0,0)$ we assume scattering by dipole plus polarization potential with [49]

$$
S_{\nu \nu^{\prime}}^{m}=\delta_{\nu \nu^{\prime}} \exp \left(-i \pi \lambda_{\nu}^{m}\right) \frac{b_{\nu}^{m}+\pi i \alpha k^{2}}{b_{\nu}^{m}-\pi i \alpha k^{2}},
$$

$$
b_{\nu}^{m}=\left(2 \lambda_{\nu}^{m}-1\right)\left(2 \lambda_{\nu}^{m}+1\right)\left(2 \lambda_{\nu}^{m}+3\right),
$$

where $\alpha$ is the isotropic part of the polarizability. Then the scattering amplitude in the fixed-nuclei approximation is

$$
\begin{aligned}
f\left(\xi_{0}, \phi_{0}, \xi, \phi\right)= & \frac{i}{k} \sum_{\nu m}\left[D_{\nu}^{m}\left(\cos \xi_{0}\right)-e^{i \pi m} S_{\nu \nu} D_{\nu}^{m}\left(-\cos \xi_{0}\right)\right] \\
& \times D_{\nu}^{m}(\cos \xi) e^{i m\left(\phi-\phi_{0}\right)},
\end{aligned}
$$

where $\xi_{0}, \xi$ are the incident and scattering polar angles, as in the previous section, and $\phi_{0}, \phi$ are corresponding azimuthal angles.

Calculating the amplitude for the transition $(00) \rightarrow(J M)$ as a function of scattering angles $\theta, \psi$ in the laboratory frame (with polar axis along the initial momentum $\mathbf{k}$, we obtain $[41,47]$

$$
f_{J M}(\theta, \psi)=\frac{2 \pi i}{k} \sum_{l l^{\prime} m} \rho_{l l^{\prime}}^{m} \tau_{J M l l^{\prime}}^{m} Y_{l M}(\theta, \psi),
$$

where

$$
\begin{aligned}
\tau_{J M l l^{\prime}}^{m}= & (-1)^{M+m}[(2 J+1)(2 l+1) / 4 \pi]^{1 / 2}\left(\begin{array}{ccc}
J & l^{\prime} & l \\
-M & 0 & M
\end{array}\right) \\
& \times\left(\begin{array}{ccc}
J & l^{\prime} & l \\
0 & -m & m
\end{array}\right), \\
& \rho_{l l^{\prime}}^{m}=\sum_{\nu}\left[1-e^{i \pi l} S_{\nu \nu}^{m}\right] a_{\nu l}^{m} a_{\nu l^{\prime}}^{m}
\end{aligned}
$$

where $a_{\nu l}^{m}$ are coefficients in expansion of dipolar harmonics, Eq. (3), in spherical harmonics, similar to expansion (9) but for arbitrary $\nu$ and $m$.

The differential cross section is calculated then as

$$
\sigma(\theta)=\sum_{J M}\left|f_{J M}(\theta, \psi)\right|^{2} .
$$

Since the amplitude $f_{11}$ is divergent as $1 / \theta$ at $\theta \rightarrow 0$, the expansion (18) converges very slowly. To speed up the convergence, we rewrite Eq. (18) in the following form [47]

$$
f_{J M}(\theta, \psi)=f_{J M}^{(B)}(\theta, \psi)+\frac{2 \pi i}{k} \sum_{l l^{\prime} m}\left(\rho_{l l^{\prime}}^{m}-\rho_{l l^{\prime}}^{m(B)}\right) \tau_{J M l l^{\prime}}^{m} Y_{l M}(\theta, \psi),
$$

where $f_{J M}^{(B)}$ is the Born amplitude [47], and $\rho_{l l^{\prime}}^{m(B)}$ is the value of $\rho_{l l^{\prime}}^{m}$ calculated in the first order in the dipole moment

$$
\begin{gathered}
\rho_{l l^{\prime}}^{m(B)}=2 \mu\left(\delta_{l^{\prime}, l-1} \frac{q_{l m}}{l}-\delta_{l^{\prime}, l+1} \frac{q_{l+1 m}}{l+1}\right), \\
q_{l m}=\left[\frac{(l-m)(l+m)}{(2 l-1)(2 l+1)}\right]^{1 / 2} .
\end{gathered}
$$

However, for substantial dipole moments the closure expression for $f_{11}$ is not sufficient. This is because the amplitude $f_{00}$ is divergent at $\theta \rightarrow 0$ as well, although only logarithmically. We prove this statement in the Appendix by calculating $f_{00}$ in the second Born approximation. Using Eqs. (A8) and (A9) 


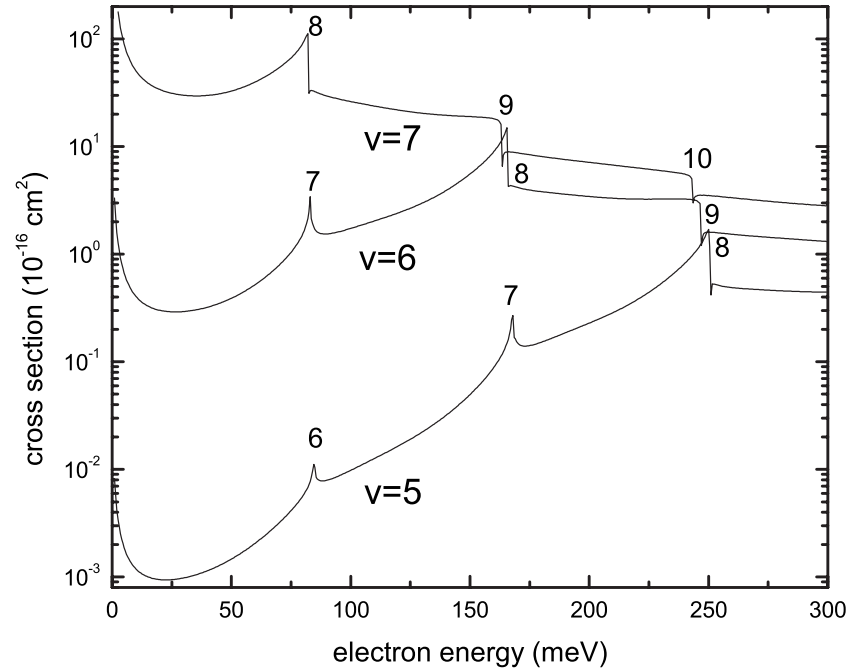

FIG. 3. DEA to vibrationally excited states of $\mathrm{CH}_{3} \mathrm{Cl}$. Numbers near the threshold peaks and/or steps indicate the VE thresholds.

from the Appendix, we can write down the closure formula for $f_{00}$ as

$$
\begin{aligned}
f_{00}(\theta)= & -\frac{2 i \mu^{2}}{3 k} \ln \left(\sin \frac{\theta}{2}\right) \\
& +\frac{i}{2 k} \sum_{l}\left(\sum_{m} \rho_{l l}^{m}-\frac{2 \mu^{2}}{3} \frac{2 l+1}{l(l+1)}\right) P_{l}(\cos \theta) .
\end{aligned}
$$

\section{RESULTS AND DISCUSSION}

\section{A. $\mathrm{CH}_{3} \mathrm{Cl}$}

VFRs in DEA for methyl halides were investigated before [5-7,50]. Here we summarize major features for further discussion using $\mathrm{CH}_{3} \mathrm{Cl}$ as an example. Adiabatic potential energy curve for the $\mathrm{CH}_{3} \mathrm{Cl}^{-}$anion supports nine weakly bound vibrational states at the $v=0$ through the $v=8$ thresholds. Only three of them, those associated with the $v=6,7$, and 8 thresholds, are noticeable in the DEA spectrum. Moreover, the positions of all resonances except the $v=8$ resonance are closer than $0.1 \mathrm{meV}$ to the corresponding thresholds. This means that the account of molecular rotations will turn them into the virtual-state cusps [51]. Figure 3 illustrates these features by showing the behavior of the DEA cross section for scattering from the $v=5,6$, and 7 states in the vicinities of the $v=6,7$, and 8 thresholds, respectively. We also observe a strong increase of cross sections with $v$ which results in strong temperature effect discussed before $[7,29,30]$.

In Fig. 4 we present $\mathrm{VE}$ of $\mathrm{CH}_{3} \mathrm{Cl}$ for a series of the $v \rightarrow v+1$ transitions. The first noticeable threshold peak appears in the $v=5 \rightarrow 6$ transition. With the further increase of $v$ the peak develops further and reaches maximum value exceeding $50 \times 10^{-16} \mathrm{~cm}^{2}$ at $v=7$. This is expected, since the $v=8$ threshold generates the most pronounced feature in the DEA cross section. An interesting shape is observed for the $v=6 \rightarrow 7$ cross section. At the $v=7$ threshold we see the virtual-state cusp, and then a broader VFR associated with

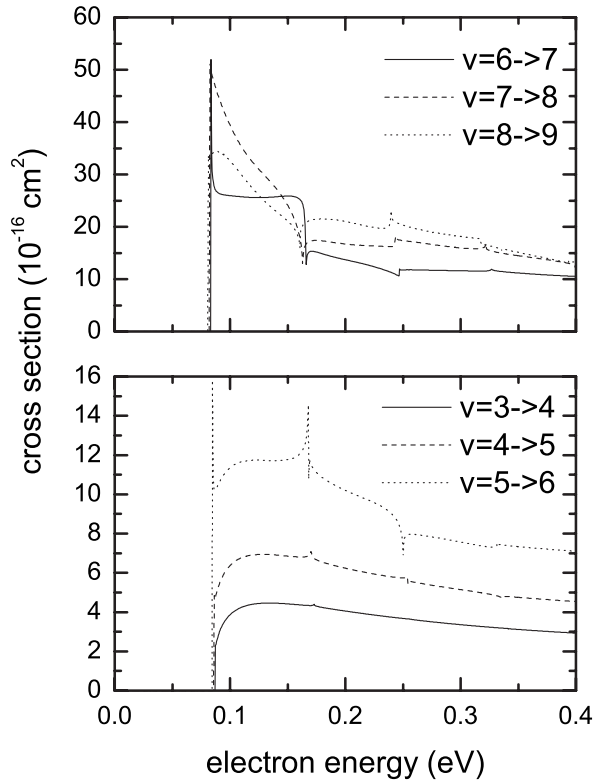

FIG. 4. VE of $\mathrm{CH}_{3} \mathrm{Cl}$ from vibrationally excited states.

the $v=8$ threshold. Cusps and step structures seen at higher thresholds are similar to those observed before in VE of hydrogen halides and $\mathrm{CH}_{3} \mathrm{I}[21,27,52,53]$.

From the above discussion it is clear that VFRs and threshold cusps can be seen in elastic cross sections only for scattering from excited states starting from $v=5$. Before presenting the corresponding cross section, we show in Fig. 5 differential elastic cross section as a function of angle for scattering from the ground vibrational state and compare it with experiment [54]. It appears that calculated cross sections are becoming too large at large scattering angles, greater than $100^{\circ}$. Ab initio complex Kohn variational calcu-

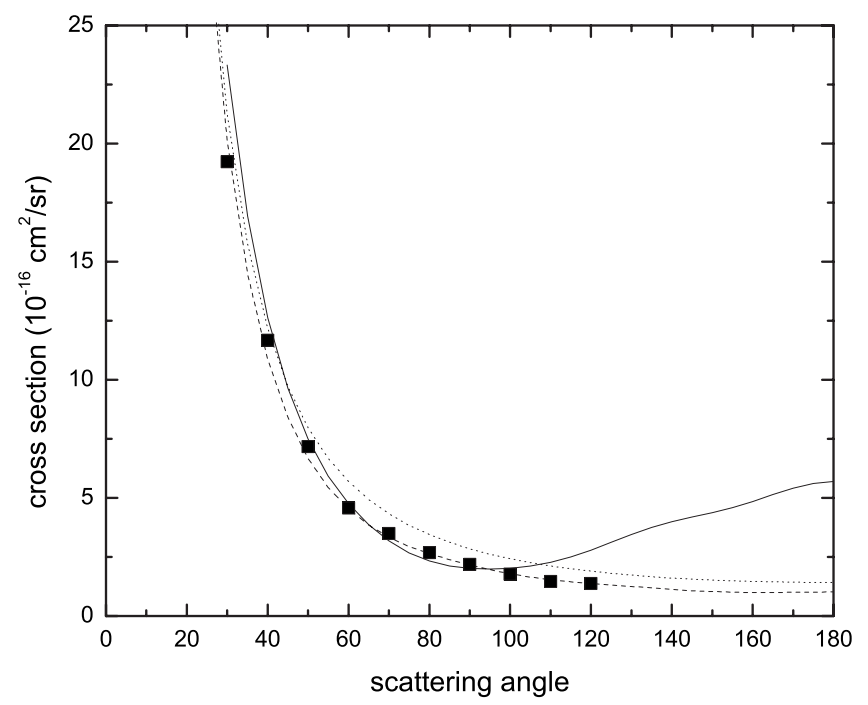

FIG. 5. Elastic scattering from the vibrationally ground state of $\mathrm{CH}_{3} \mathrm{Cl}$ as a function of the scattering angle at $E=0.5 \mathrm{eV}$. Solid line: present calculation with full inclusion of polarization. Dashed line: calculation with no polarization included in the partial wave $\nu=1$. Dotted line: dipole-Born approximation. Squares: experimental data [54]. 
lations [55] do not exhibit growth at higher scattering angles. The partial wave analysis of our cross section indicates that our method perhaps overestimates the polarization contribution to the scattering matrix for $\nu=1$ (which approximately corresponds to $l=1)$. Indeed, Eq. (15) for the scattering matrix is valid if the polarization potential can be considered as a perturbation compared to the centrifugal potential, similar to the equation of O'Malley et al. [56]. For the polarizability of $\mathrm{CH}_{3} \mathrm{Cl}, \alpha=31$ a.u., and $E=0.5 \mathrm{eV}, l=1$ this is becoming a very crude approximation. To check this we also performed another calculation whereby the polarization contribution for $\nu=1$ was neglected. Agreement with experiment [54] and with the complex Kohn calculations [55] becomes much better, which is perhaps somewhat fortuitous, but this indicates the important role of the $p$ scattering by the polarization potential even at such a low energy as $0.5 \mathrm{eV}$. Apparently this is an upper bound for our resonant approach which does not employ accurate methods for calculation of contribution of higher partial waves. Therefore in our further discussion of elastic scattering we will consider only energies below $0.5 \mathrm{eV}$. We should add, however, that our method gives reliable results at higher energies for resonance processes such as DEA and VE.

Another important observation following from Fig. 5 is the high sensitivity of the cross section to the polarization contribution even at relatively small angles about $30^{\circ}$ where the conventional wisdom says that the dipolar interaction should dominate. Full inclusion of polarization according to Eq. (15) gives the result which is higher than the dipole Born approximation, whereas elimination of polarization in Eq. (15) at $\nu=1$ gives lower cross section which is closer to the experimental value. We conclude that inaccurate inclusion of polarization can lead to an overestimation of the cross section at low angles. This might be relevant to disagreement at low angles between the complex Kohn calculations and experiment discussed in Ref. [55].

In Fig. 6 we present low-energy elastic differential cross sections for $\mathrm{CH}_{3} \mathrm{Cl}$ at $\theta=100^{\circ}$ and compare them with the dipole-Born results. The present calculations give cross sections which are much smaller than the Born results above the first VE threshold, but they increase significantly towards smaller energies. Pronounced step structures and cusps are observed at the $v=7$ and $v=8$ thresholds.

\section{B. $\mathrm{CH}_{3} \mathrm{Br}$}

In $\mathrm{CH}_{3} \mathrm{Br}$ there are five dipole-supported states associated with the $v=0$ through $v=4$ thresholds [7]. Only the highest $v=4$ feature appears as a pronounced VFR. Its existence was recently confirmed by experimental observations [50], where the integrated VE cross sections for $\mathrm{CH}_{3} \mathrm{Br}$ were also calculated. Threshold peaks appear even in the $v=0 \rightarrow 1$ transition and become more pronounced for higher $v$. The largest cross section, $v=2 \rightarrow 3$ reaches very high value at the peak, $96 \times 10^{-16} \mathrm{~cm}^{2}$, not very far from its unitary limit, $158 \times 10^{-16} \mathrm{~cm}^{2}$. This is perhaps the largest VE cross section ever predicted.

In Fig. 7 we show VE cross sections at two angles and compare them with those from the direct dipole contribution.

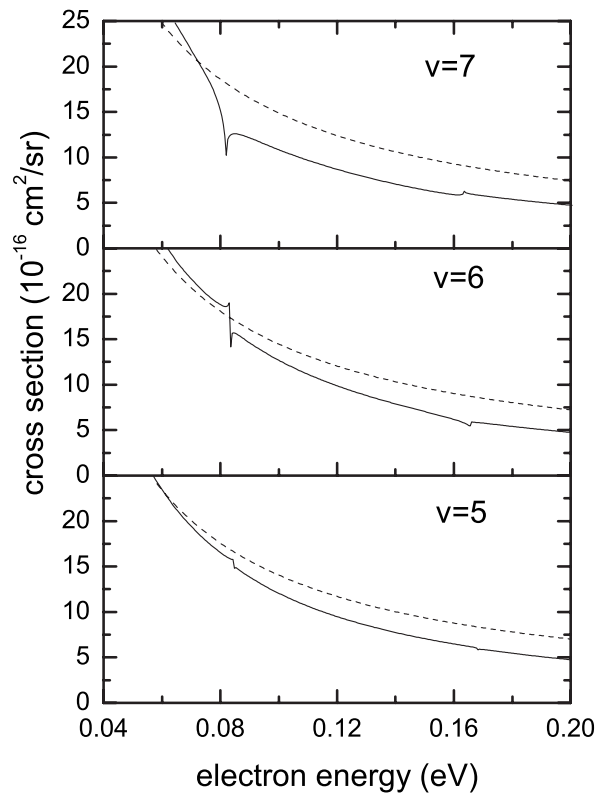

FIG. 6. Elastic scattering from vibrationally excited states of $\mathrm{CH}_{3} \mathrm{Cl}$ at $\theta=100^{\circ}$. Solid lines: present calculations. Dashed lines: dipole-Born approximation.

The latter becomes more important at lower angles, although in the case of the $v=2 \rightarrow 3$ transition the resonant process strongly dominates even at $\theta=30^{\circ}$. Another interesting feature is a peculiar shape of the $2 \rightarrow 3$ cross section. It is dominated by the virtual-state effect just above the $v=3$ threshold and VFR below the $v=4$ threshold, and is similar to the $v=6 \rightarrow 7$ excitation in $\mathrm{CH}_{3} \mathrm{Cl}$ presented in Fig. 4 .

In Fig. 8 we present elastic differential cross sections for scattering from the $v=2$ and $v=3$ states and compare them with the dipole-Born result. At $\theta=30^{\circ}$ both curves are quite close to each other, whereas a noticeable difference is observed for $\theta=100^{\circ}$.

\section{C. $\mathrm{CH}_{3} \mathrm{I}$}

In $\mathrm{CH}_{3} \mathrm{I}$ there are two weakly bound states associated with $v=0$ and $v=1$ thresholds. The latter appears as pronounced VFR in DEA [6,7]. Since the dipole moment of $\mathrm{CH}_{3} \mathrm{I}$ is relatively weak, the inclusion of polarization is becoming crucial for obtaining the correct shape of VFR [57]. The total VE cross section and the resonance contribution to the elastic cross section was compared to experimental data at $\theta=135^{\circ}$ in Ref. [27]. Here we show a more direct comparison by calculating differential cross sections at $135^{\circ}$.

In Fig. 9 we present $v=0 \rightarrow 1$ and $v=1 \rightarrow 2$ cross sections and compare them with arbitrary normalized experimental data of Allan [27]. Qualitatively all of the main features in the experimental cross section are reproduced by theory, although the theoretical features (threshold peak and structures at higher thresholds) are sharper, apparently due to limited experimental energy resolution. Due to substantial population of the first excited vibrational state in experiment (about $8 \%$ ), the shape of the experimental cross section is apparently affected by the $v=1 \rightarrow 2$ transition. Overall, the present comparison does not differ noticeably from that presented in 


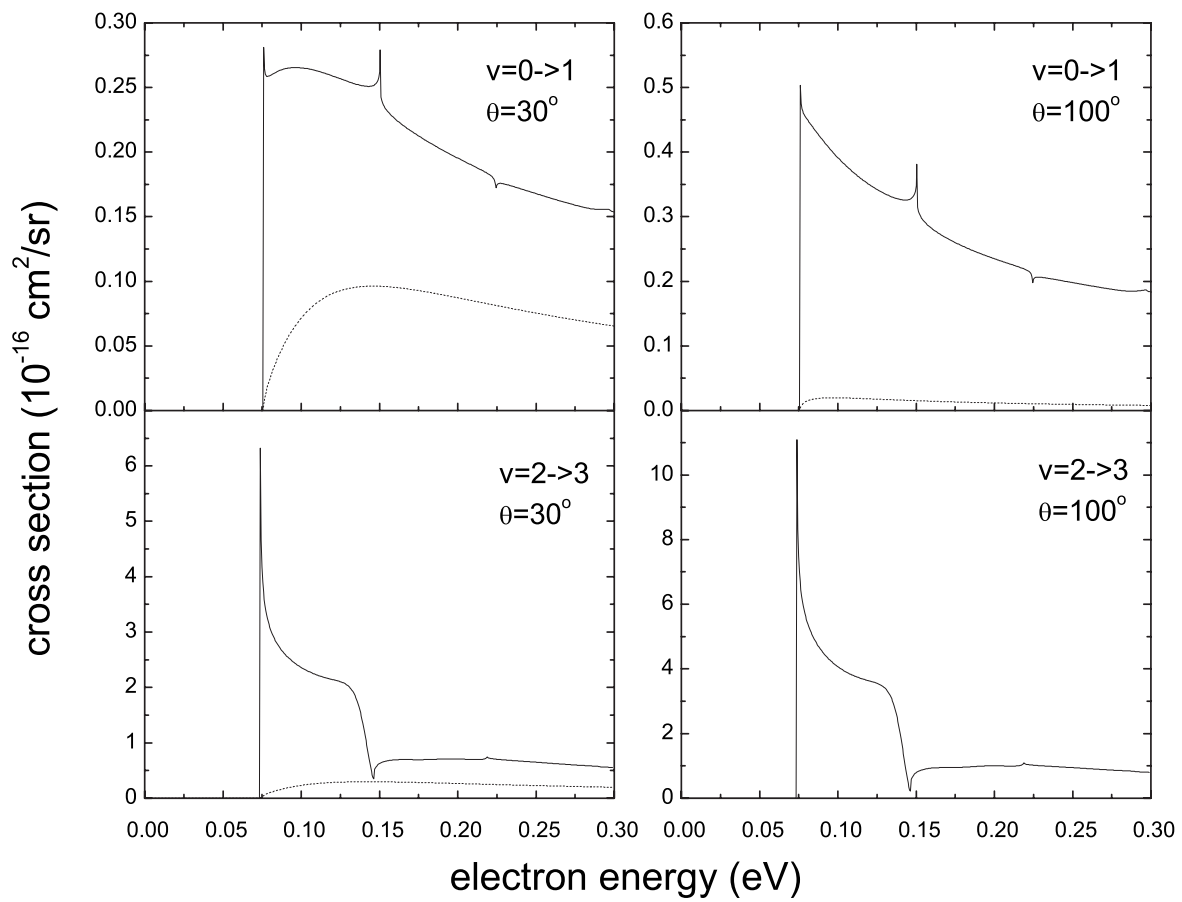

FIG. 7. Differential cross sections for $\mathrm{VE}$ of $\mathrm{CH}_{3} \mathrm{Br}$. Solid lines: present calculations. Dotted lines: dipole-Born approximation.
Ref. [27] since the energy dependence of the integrated cross section and differential cross section at large angles is very similar. Note that the direct contribution is very small in this case and its peak value is $0.00324 \times 10^{-16} \mathrm{~cm}^{2} / \mathrm{sr}$ at $E=0.081 \mathrm{eV}$.

In Fig. 10 we present elastic differential cross section at $\theta=135^{\circ}$. In contrast to the $\mathrm{CH}_{3} \mathrm{Br}$ case, the present cross section differs substantially from the dipole-Born contribution, apparently because of the smaller dipole moment and larger polarizability in this case $(\alpha=54$ a.u.). The accurate account of polarization becomes important even at relatively low energy. In order to check the polarization effect, we, as in the case of $\mathrm{CH}_{3} \mathrm{Cl}$, performed additional calculation without inclusion of polarization in the $p$ wave. The cross section has become even higher. The difference with the first calculation represents the uncertainty of our results in the present case. With regard to comparison with experiment, the experimental cross section grows not as fast as the calculated when approaching the zero energy. The disagreement becomes particularly striking below the excitation threshold, partly because of the rapidly deteriorating quality of the incident electron beam [27]. The calculated dip at the excitation threshold is more pronounced than experimental. Partly this can be explained by the neglect of coupling with other vibrational

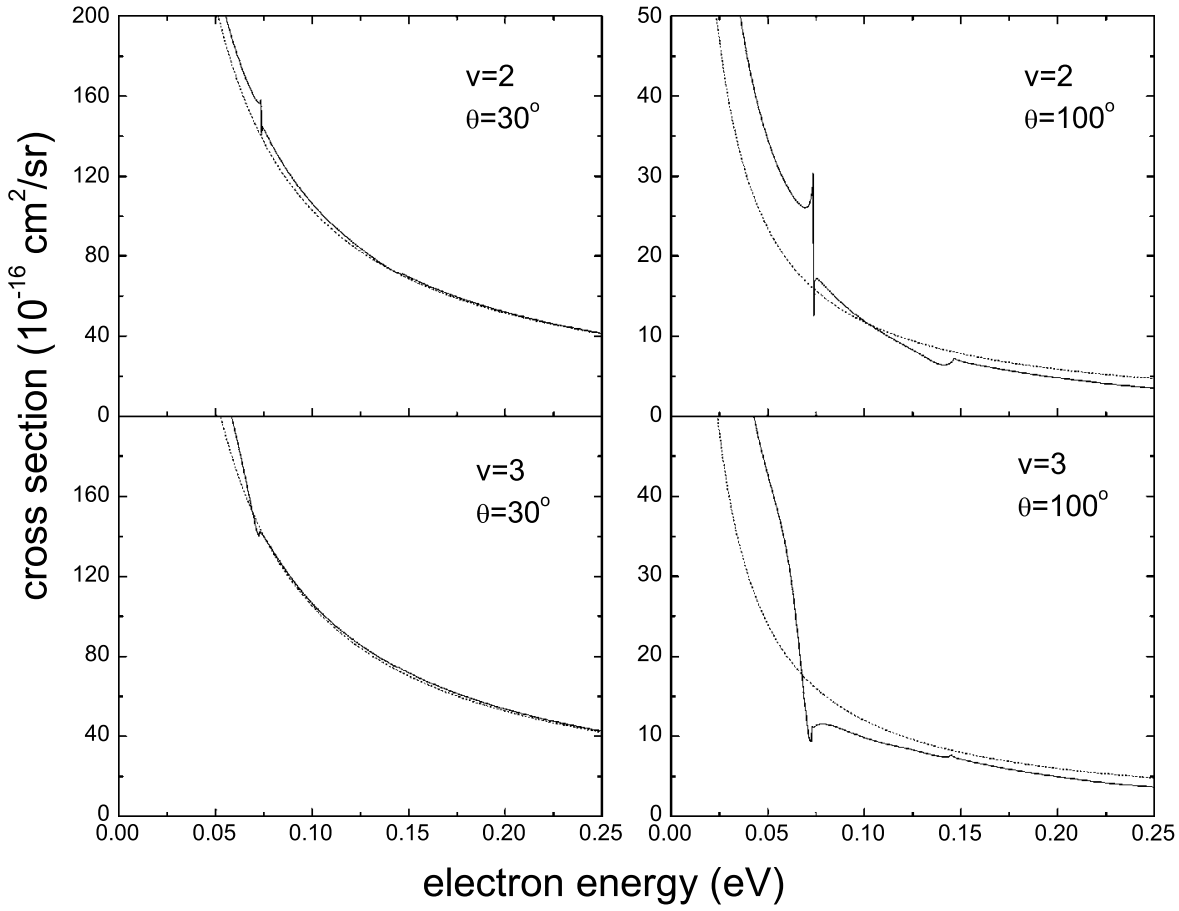

FIG. 8. Elastic scattering from vibrationally excited states of $\mathrm{CH}_{3} \mathrm{Br}$ at $\theta=30$ and $100^{\circ}$. Solid lines: present calculations. Dotted lines: dipole-Born approximation. 


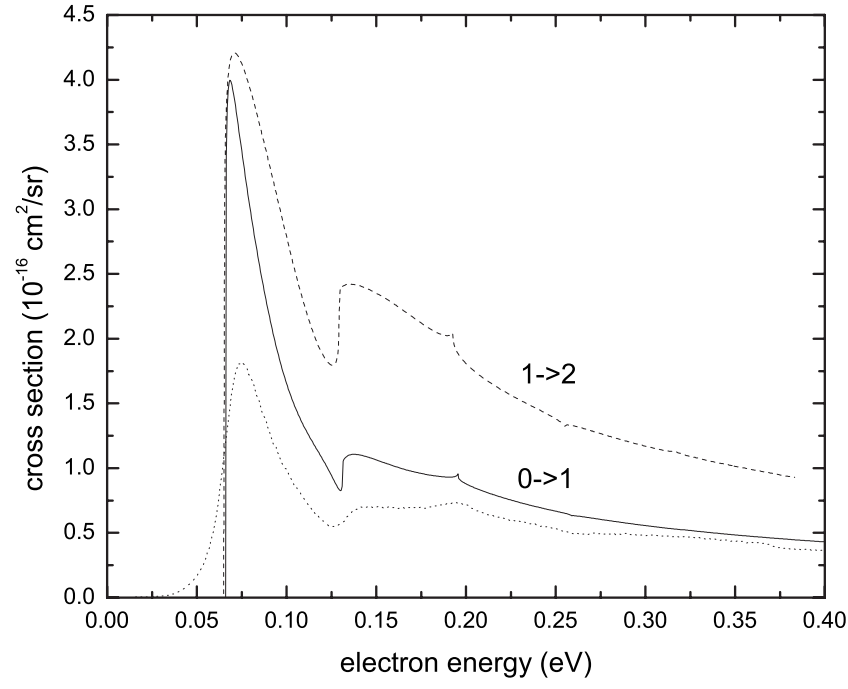

FIG. 9. VE of $\mathrm{CH}_{3} \mathrm{I}$ at $\theta=135^{\circ}$. Solid and dashed lines: present calculations. Dotted line: arbitrarily normalized experimental data [27].

modes. On the other hand, the theory reproduces very well the shape of VFR in DEA channel [6], therefore obviously there could be several reasons for disagreement. We conclude that the model employed in the present paper is much better suited for studies of resonance processes, whereas calculation of elastic scattering requires more accurate description of the direct process and higher partial waves.

Finally, in Fig. 11 we present comparison of the integrated resonance contribution to the elastic scattering with the total integrated resonant cross section including DEA and VE cross sections. We observe that the feature at the $v=1$ threshold is strongly suppressed in the total cross section. This might be relevant to observation of VFR in uracil [23], where pronounced VFR is observed in the DEA channel

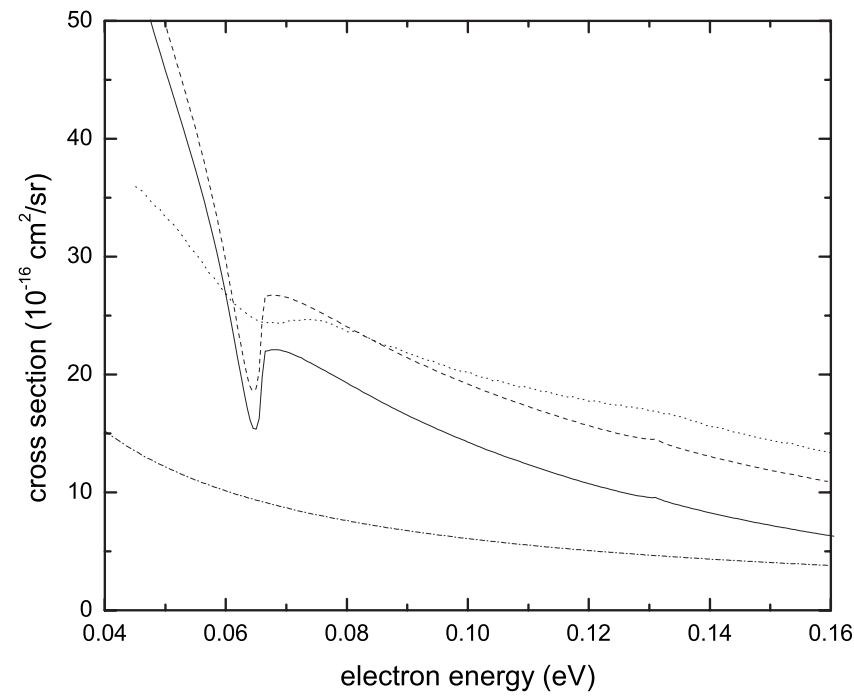

FIG. 10. Elastic scattering from $\mathrm{CH}_{3} \mathrm{I}$ at $\theta=135^{\circ}$. Solid line: present calculation with full inclusion of polarization. Dashed line: calculation with no polarization included in the partial wave $\nu=1$. Dash-dotted line: dipole-Born approximation. Dotted line: experimental data [27].

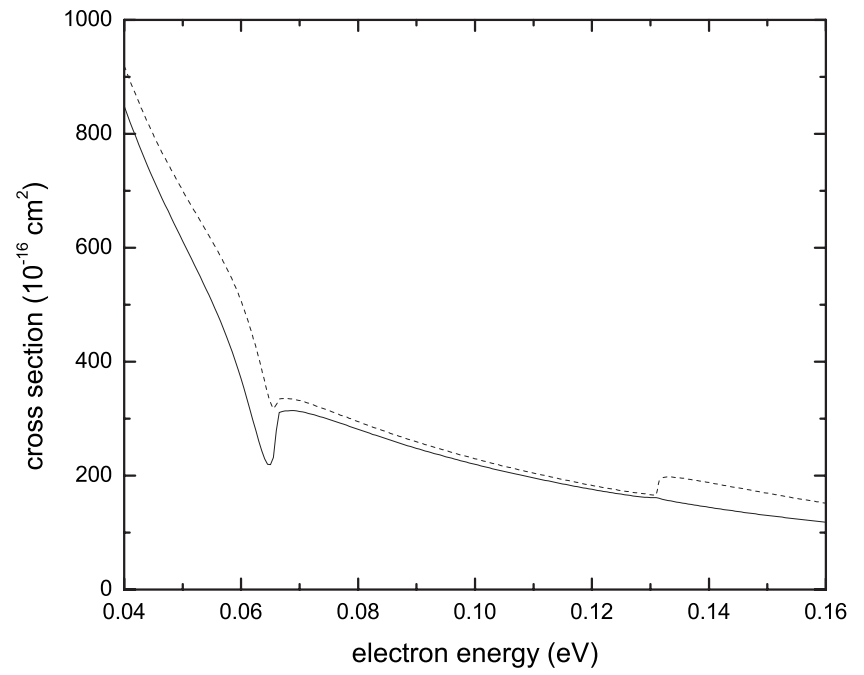

FIG. 11. Angular-integrated cross sections for scattering from $\mathrm{CH}_{3}$ I. Solid line: resonance contribution to elastic scattering. Dashed line: total resonance contribution including VE and DEA.

whereas measurements of the total cross section by the use of the electron transmission spectroscopy technique do not give any visible feature at the $v=1$ threshold.

\section{CONCLUSION}

We have presented analysis of the resonance and threshold effects in elastic and inelastic scattering of electrons by methyl halides. The results of this paper are of general significance due to the importance of VFRs in many systems including biological molecules and positron-containing systems.

In contrast to collisions with hydrogen halides, studied in Refs. [21,28,52,53], where the DEA process is endothermic (with the exception of $\mathrm{HI}$ ), collisions with methyl halides involve the DEA process which is exothermic. In spite of the possibility of predissociation after capture in a dipole supported state, VFRs in methyl halides can have narrow width. Moreover, for the dipole-supported states with very small binding energies (below $0.1 \mathrm{meV}$ ), both contributions to the resonance width, due to autodetachment and predissociation, become so small that from the practical point of view the resonance disappears completely. This is pertinent to resonances in $\mathrm{CH}_{3} \mathrm{Cl}$ with $v<6$ and in $\mathrm{CH}_{3} \mathrm{Br}$ with $v<3$.

Therefore, in spite of the supercritical value of the dipole moment of $\mathrm{CH}_{3} \mathrm{Cl}$ and $\mathrm{CH}_{3} \mathrm{Br}$, no noticeable threshold features are observed in scattering by $\mathrm{CH}_{3} \mathrm{Cl}(v)$ for $v<6$ and by $\mathrm{CH}_{3} \mathrm{Br}(v)$ for $v<3$. We should emphasize that this conclusion has nothing to do with rotational motion of the molecule. Of course, if we are interested in energies very close to the threshold (so that the distance to the threshold is comparable to the rotational spacing), the rotational motion should be taken into account. In this case VFRs with binding energies below the rotational spacing will be converted into virtual states [51], but all major features in the cross sections will remain unaffected even with the best energy resolution (about $1 \mathrm{meV}$ ) available in current experiments. 
In the $\mathrm{CH}_{3} \mathrm{I}$ molecule the only VFR is associated with the $v=1$ threshold. This makes it easier for observations. Indeed, the pronounced features were observed in DEA [6] and VE [27] cross sections, and, somewhat less pronounced in the elastic scattering [27]. Our predictions for VE and elastic scattering from excited states of $\mathrm{CH}_{3} \mathrm{Cl}$ and $\mathrm{CH}_{3} \mathrm{Br}$ will await for experimental confirmation. It should be stressed that recent observation [50] of VFRs in DEA to $\mathrm{CH}_{3} \mathrm{Br}$ gives us confidence in reliability of our results for this molecule.

\section{ACKNOWLEDGMENTS}

The authors are grateful to H. Hotop for many stimulating discussions. This work has been supported by the National Science Foundation through Grant No. PHY-0354688.

\section{APPENDIX: ROTATIONALLY ELASTIC SCATTERING AMPLITUDE}

We are interested here in rotationally elastic amplitude for a diatomic molecule and its convergence at $\theta \rightarrow 0$. For simplicity we will consider the elastic scattering amplitude $f_{00}$ for pure dipolar scattering and will obtain its explicit form in the second Born approximation. This will prove its divergence at $\theta \rightarrow 0$ and will allow us to derive the closure expansion (25) for the rotationally elastic scattering case.

For $J=0$, Eq. (18) becomes

$$
\begin{gathered}
f_{00}(\theta)=\frac{i}{2 k} \sum_{l m} \rho_{l l}^{m} P_{l}(\cos \theta), \\
\rho_{l l}^{m}=\sum_{\nu}\left(1-\exp \left\{i \pi\left[l-\lambda_{\nu}^{m}\right]\right\}\right)\left[a_{\nu l}^{m}\right]^{2},
\end{gathered}
$$

where we use $S_{\nu \nu}^{m}=\exp \left(-i \pi \lambda_{\nu}^{m}\right)$ for a pure dipole potential. Strictly speaking, this equation requires a modification for a supercritical dipole moment and $\nu=0$, but for the present case this is not relevant since we are concerned with the high- $l$ behavior.

We will expand now $\rho_{l l}^{m}$ in powers of $\mu$ and will keep only the first nonvanishing term, that is the term proportional to $\mu^{2}$. First we obtain the coefficients $a_{\nu l}^{m}$ and the eigenvalues $\lambda_{\mu}^{m}$ in the first nonvanishing order

$$
\begin{gathered}
a_{\nu l}^{m}=\delta_{\nu l}+\mu\left(-\delta_{\nu, l-1} \frac{q_{l m}}{l}+\delta_{\nu, l+1} \frac{q_{l+1 m}}{l+1}\right), \\
\lambda_{\nu}^{m}=\nu+\frac{2 \mu^{2}}{2 \nu+1}\left(\frac{q_{\nu m}^{2}}{\nu}-\frac{q_{\nu+1 m}^{2}}{\nu+1}\right),
\end{gathered}
$$

where $q_{l m}$ is given by Eq. (24). From Eq. (A2)

$$
\begin{aligned}
\rho_{l l}^{m}= & 1-\exp \left[i \pi\left(l-\lambda_{l}^{m}\right)\right]+\left\{1-\exp \left[i \pi\left(l-\lambda_{l-1}^{m}\right)\right]\right\}\left(a_{l-1 l}^{m}\right)^{2} \\
& +\left\{1-\exp \left[i \pi\left(l-\lambda_{l+1}^{m}\right)\right]\right\}\left(a_{l+1 l}^{m}\right)^{2} .
\end{aligned}
$$

Using Eqs. (A3) and (A4), we obtain from here

$$
\rho_{l l}^{m}=\frac{2 \pi i \mu^{2}}{2 l+1}\left(\frac{q_{l m}^{2}}{l}-\frac{q_{l+1 m}^{2}}{l+1}\right)+2 \mu^{2}\left(\frac{q_{l m}^{2}}{l^{2}}+\frac{q_{l+1 m}^{2}}{(l+1)^{2}}\right) .
$$

For summation over $m$ we use the equation

$$
\sum_{m=-l}^{l} q_{l m}^{2}=\frac{1}{(2 l-1)(2 l+1)} \sum_{m=-l}^{l}(l-m)(l+m)=\frac{l}{3} .
$$

This leads to the result

$$
\sum_{m=-l}^{l} \rho_{l l}^{m}=\frac{2 \mu^{2}}{3}\left(\frac{1}{l}+\frac{1}{l+1}\right)
$$

Note that imaginary part of $\rho_{l l}^{m}$ turns to 0 in the second order in $\mu$.

Finally, summation over $l$ gives the following expression for the scattering amplitude:

$$
f_{00}(\theta)=-\frac{2 i \mu^{2}}{3 k} \ln \left(\sin \frac{\theta}{2}\right)
$$

We conclude that the imaginary part of the rotationally elastic scattering amplitude diverges logarithmically in the forward direction. This divergence is consistent with the optical theorem since the total integrated cross section is divergent due to the $J \rightarrow J \pm 1$ transitions. On the other hand, since the divergence is only logarithmical, the integrated cross section for the rotationally elastic cross section is finite.
[1] D. Klar, M.-W. Ruf, and H. Hotop, Chem. Phys. Lett. 189, 448 (1992).

[2] D. Klar, M.-W. Ruf, and H. Hotop, Meas. Sci. Technol. 5, 1248 (1994).

[3] S. V. Hoffmann, S. L. Lunt, N. C. Jones, D. Field, and J.-P. Ziesel, Rev. Sci. Instrum. 73, 4157 (2002).

[4] J. P. Ziesel, N. C. Jones, D. Field, and L. B. Madsen, Phys. Rev. Lett. 90, 083201 (2003).

[5] H. Hotop, M.-W. Ruf, M. Allan, and I. I. Fabrikant, Adv. At., Mol., Opt. Phys. 49, 85 (2003).

[6] A. Schramm, I. I. Fabrikant, J. M. Weber, E. Leber, M.-W. Ruf, H. Hotop, J. Phys. B 32, 2153 (1999).
[7] R. S. Wilde, G. A. Gallup, and I. I. Fabrikant, J. Phys. B 33, 5479 (2000).

[8] D. Klar, M.-W. Ruf, I. I. Fabrikant, and H. Hotop, J. Phys. B 34, 3855 (2001).

[9] A. Schramm, M.-W. Ruf, M. Stano, S. Matejcik, I. I. Fabrikant, and H. Hotop, J. Phys. B 35, 4179 (2002).

[10] I. I. Fabrikant and H. Hotop, Phys. Rev. Lett. 94, 063201 (2005).

[11] I. I. Fabrikant, H. Hotop, and M. Allan, Phys. Rev. A 71, 022712 (2005).

[12] S. Marienfeld, I. I. Fabrikant, M. Braun, M.-W. Ruf and H. Hotop, J. Phys. B 39, 105 (2006). 
[13] S. Marienfeld, T. Sunagawa, I. I. Fabrikant, M. Braun, M.-W. Ruf, and H. Hotop, J. Chem. Phys. 124, 154316 (2006).

[14] R. Čurik, J. P. Ziesel, N. C. Jones, T. A. Field, and D. Field, Phys. Rev. Lett. 97, 123202 (2006).

[15] M. Külz, M. Keil, A. Kortyna, B. Schellhaass, J. Hauck, K. Bergmann, W. Meyer, and D. Weyh, Phys. Rev. A 53, 3324 (1996).

[16] A. Rosa, F. Brüning, S. V. K. Kumar, and E. Illenberger, Chem. Phys. Lett. 391, 361 (2004).

[17] M. Braun, F. Gruber, M.-W. Ruf, S. V. K. Kumar, E. Illenberger and H. Hotop, Chem. Phys. 329, 148 (2006).

[18] W. Domcke and L. S. Cederbaum, J. Phys. B 14, 149 (1981).

[19] J.-P. Gauyacq and A. Herzenberg, Phys. Rev. A 25, 2959 (1982).

[20] G. Knoth, M. Gote, M. Rädle, K. Jung, and H. Ehrhardt, Phys. Rev. Lett. 62, 1735 (1989).

[21] M. Čižek, J. Horáček, M. Allan, I. I. Fabrikant, and W. Domcke, J. Phys. B 36, 2837 (2003).

[22] M. Allan and T. Skalický, J. Phys. B 36, 3397 (2003).

[23] A. M. Scheer, K. Aflatooni, G. A. Gallup, and P. D. Burrow, Phys. Rev. Lett. 92, 068102 (2004)

[24] P. D. Burrow, G. A. Gallup, A. M. Scheer, S. Denifl, S. Ptasinska, T. Märk, and P. Scheier, J. Chem. Phys. 124, 124310 (2006).

[25] L. D. Barnes, S. J. Gilbert, and C. M. Surko, Phys. Rev. A 67, 032706 (2003).

[26] G. F. Gribakin and C. M. R. Lee, Phys. Rev. Lett. 97, 193201 (2006).

[27] M. Allan and I. I. Fabrikant, J. Phys. B 35, 1025 (2002).

[28] J. Horáček, M. Čižek and W. Domcke, Theor. Chem. Acc. 100, 31 (1998).

[29] I. I. Fabrikant, J. Phys. B 27, 4325 (1994).

[30] D. M. Pearl, P. D. Burrow, I. I. Fabrikant, and G. A. Gallup, J. Chem. Phys. 102, 2737 (1995).

[31] CRC Handbook of Chemistry and Physics, edited by D. R. Lide (CRC Press, Boca Raton, 2004).

[32] D. M. Bishop and L. M. Cheung, J. Phys. Chem. Ref. Data 11, 119 (1982).

[33] I. I. Fabrikant, Phys. Rev. A 43, 3478 (1991).

[34] J. F. Ogilvie, W. R. Rodwell, and R. H. Tipping, J. Chem. Phys. 73, 5221 (1980).
[35] G. A. Gallup, R. L. Vance, J. R. Collins, and J. M. Norbeck, Adv. Quantum Chem. 16, 229 (1982).

[36] G. A. Gallup, Valence Bond Methods (Cambridge University Press, Cambridge, 2002).

[37] M. W. Schmidt et al., J. Comput. Chem. 14, 1347 (1993).

[38] M. H. Mittleman and R. E. von Holdt, Phys. Rev. 140, A726 (1965).

[39] I. I. Fabrikant, Sov. Phys. JETP 44, 77 (1976).

[40] K. Takayanagi, Suppl. Prog. Theor. Phys. 40, 216 (1967).

[41] I. I. Fabrikant, Phys. Lett. 77A, 421 (1980).

[42] O. H. Crawford, J. Chem. Phys. 47, 1100 (1967).

[43] P. C. Engelking, Phys. Rev. A 26, 740 (1982).

[44] P. C. Engelking and D. R. Herrick, Phys. Rev. A 29, 2425 (1984).

[45] G. Herzberg, Infrared and Raman Spectra of Polyatomic Molecules (Van Nostrand, New York, 1945).

[46] M. H. Mittleman, J. L. Peacher, and B. F. Rozsnyai, Phys. Rev. 176, 180 (1968).

[47] I. I. Fabrikant, J. Phys. B 16, 1253 (1983).

[48] I. Shimamura, Phys. Rev. A 23, 3350 (1981).

[49] I. I. Fabrikant, J. Phys. B 12, 3599 (1979).

[50] M. Braun, I. I. Fabrikant, M.-W. Ruf, and H. Hotop, J. Phys. B 40, 659 (2007).

[51] I. I. Fabrikant and R. S. Wilde, J. Phys. B 32, 235 (1999).

[52] A.-Ch. Sergenton, L. Jungo, and M. Allan, Phys. Rev. A 61, 062702 (2000)

[53] M. Čižek, J. Horáček, A.-Ch. Sergenton, D. B. Popović, M. Allan, W. Domcke, T. Leininger, and F. X. Gadea, Phys. Rev. A 63, 062710 (2001).

[54] X. Shi, V. K. Chan, G. A. Gallup, and P. D. Burrow, J. Chem. Phys. 104, 1855 (1996).

[55] T. N. Rescigno, A. E. Orel, and C. W. McCurdy, Phys. Rev. A 56, 2855 (1997).

[56] T. F. O’Malley, L. Spruch, and L. Rosenberg, J. Math. Phys. 2, 491 (1961).

[57] E. Leber, I. I. Fabrikant, J. M. Weber, M.-W. Ruf, and H. Hotop, in Dissociative Recombination: Theory, Experiment and Applications IV, edited by M. Larsson, J. B. A. Mitchell, and I. F. Schneider (World Scientific, Singapore, 2000), pp. 69-76. 